Images du travail, travail des images

$10 \mid 2021$

La fiction au travail

\title{
La fiction au travail
}

Recherche et formation à la recherche au moyen d'images de fiction (cinéma, télévision ou Web)

Pascal Cesaro et Pierre Fournier

\section{(2) OpenEdition}

\section{Journals}

Édition électronique

URL : https://journals.openedition.org/itti/1533

DOI : $10.4000 /$ itti. 1533

Éditeur

Université de Poitiers

\section{Référence électronique}

Pascal Cesaro et Pierre Fournier, «La fiction au travail », Images du travail, travail des images [En ligne],

10 | 2021, mis en ligne le 20 février 2021, consulté le 24 janvier 2022. URL : http://

journals.openedition.org/itti/1533; DOI : https://doi.org/10.4000/itti.1533

Ce document a été généré automatiquement le 24 janvier 2022.

Images du travail, travail des images 


\title{
La fiction au travail
}

\author{
Recherche et formation à la recherche au moyen d'images de fiction \\ (cinéma, télévision ou Web)
}

Pascal Cesaro et Pierre Fournier

1 Depuis les débuts du cinéma, les images animées servent à raconter des histoires inventées, des fictions, autant qu'à livrer des chroniques du réel. La magie du cadrage, des jeux d'échelles, du montage fournit des possibilités formidables pour donner corps à des fictions produites par l'imagination autant que la caméra peut tenter de répliquer le réel avec détails dans un projet documentaire soucieux d'authenticité. À première vue, seul le second usage de l'image intéresse les sciences sociales: en intégrant la caméra au processus de recherche ou en utilisant le film comme objet d'étude, pour la part de vérité du projet documentaire, mais aussi pour la réflexion critique à conduire sur la communication de masse quand le reportage audiovisuel prend place dans un projet d'information journalistique, l'occasion d'analyser les points de vue qui s'expriment sous couvert de restitution objective du réel. Mais cette opposition entre le vrai et le faux peut paraître dépassée à l'ère de « l'esthétique » pour définir l'objet film : tout documentaire met en jeu une fiction particulière et, réciproquement, toute fiction inscrit son tournage dans le réel. En réalité, c'est cet écart entre le référent et son image qui constitue la base de tout récit, et la fiction comme le documentaire peuvent raconter cet écart. La fiction cinématographique fournit donc une matière considérable à plusieurs disciplines des sciences sociales. On pense en premier à Jean Rouch pour qui le cinéma était d'abord un moyen de contourner des problèmes posés par la méthode ethnographique, comme avec le film La Punition où il utilise la séduction pour réaliser des entretiens au service à la fois de l'enquête sociologique et de sa pratique cinématographique. 
Image 1 : Nadine Ballot et Jean-Claude Darnal jouent la séduction entre Nadine et JeanClaude dans La Punition

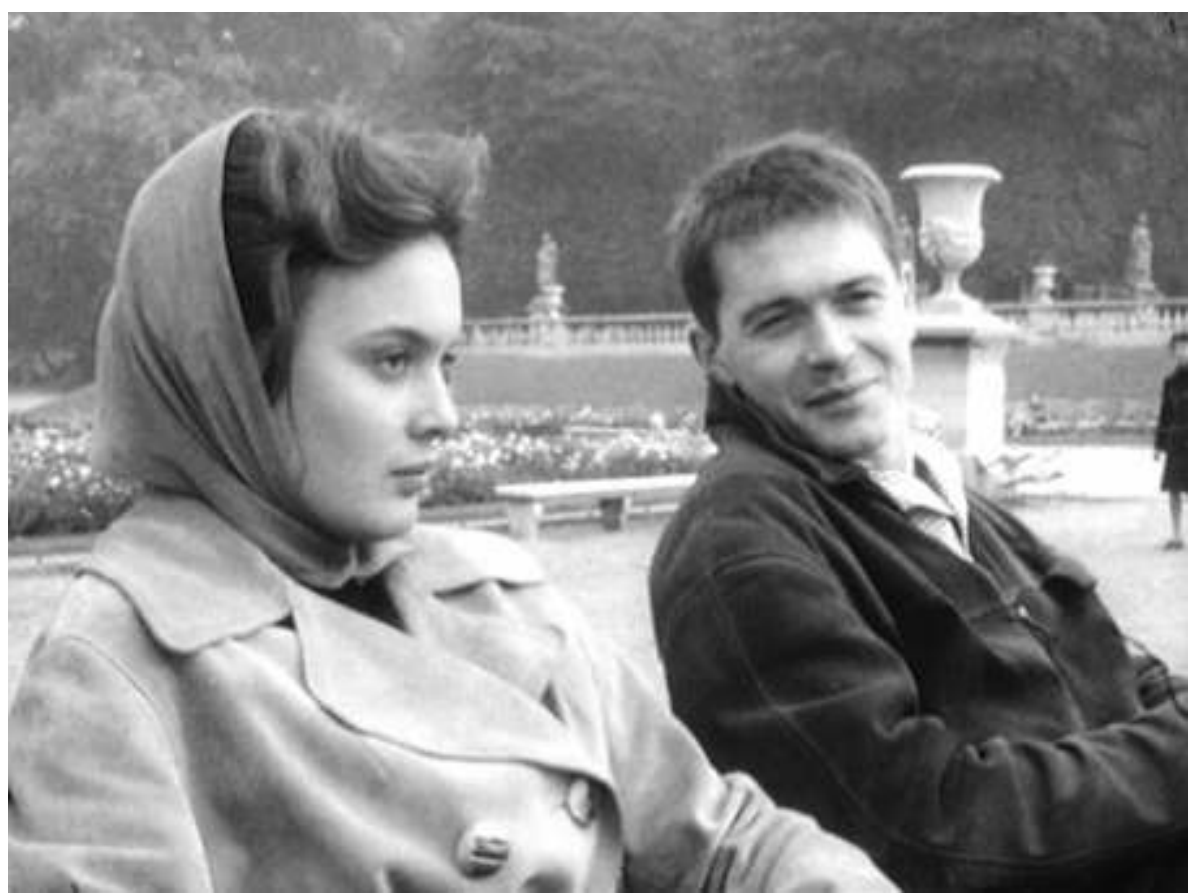

Photogramme extrait de La Punition, un film de Jean Rouch (1962), copie d'écran. Visionner un extrait du film La Punition.

\section{Fiction et recherche : une longue histoire}

2 La fiction cinématographique est bien sûr centrale pour l'histoire des techniques à travers les techniques de figuration du réel : les musées du cinéma et de la télévision en témoignent. C'est aussi le cas pour l'histoire culturelle : elle s'emploie à circonscrire à travers les fictions les domaines du réel qu'une société décide de symboliser et revendique comme tels au fil des conjonctures. C'est plus indirectement qu'intervient la fiction dans le cas de l'anthropologie. Le premier mouvement va plutôt dans le sens d'un recours à la fonction documentaire de l'image. De longue date, les disciplines des sciences sociales recourant à l'ethnographie, à l'observation directe de situations d'interaction sociale, valorisant la compréhension permise par la proximité voire le partage d'expérience avec les personnes étudiées, cherchent à faire usage de la caméra dans la phase de recueil des données au plus près des situations investiguées. Il s'agit de pouvoir retravailler ces observations à distance du terrain et de dépasser par là la division du travail entre collecte sur place et analyse en cabinet, voire la division entre les disciplines de l'ethnologie et de l'anthropologie. Il s'agit aussi de pouvoir revisionner à l'envi pour surmonter la modestie de nos capacités d'attention dans l'instant et pour écarter notre regard sur ce qui n'est pas au centre de l'action. Au-delà, au moment d'analyser le film ethnographique, un troisième usage des images collectées apparaît parfois : proposer à certains enquêtés de les commenter dans une démarche de confrontation à l'image de soi dans l'espoir d'accéder à une désingularisation des observations réalisées, à l'explicitation des arrière-plans de l'action (Theureau, 2010). Enfin, le projet de les utiliser à titre d'illustration au moment de présenter des résultats de recherche vise à les montrer au lecteur comme attestation du réel permettant de 
juger de la justesse de l'analyse ou tout au moins de son respect scrupuleux du matériau empirique collecté.

3 Toutefois, les ressources de la narration fictionnelle sont souvent mobilisées par l'anthropologue au moment de partager les images du terrain avec un public formé au spectacle cinématographique et télévisuel : pour favoriser des effets de réel ouvrant à une compréhension de l'humanité et de la différence en son sein. La fiction audiovisuelle a donc, depuis très longtemps, partie liée avec la recherche en sciences sociales, comme source et comme ressource. Rouvrir cette question de la fiction audiovisuelle comme ressource pour la recherche s'impose à l'heure où la recherche cinématographique circonscrit mieux les mécanismes de la narration fictionnelle qui sont propres au divertissement audiovisuel, à l'heure où l'accès aux images de fiction consacrée, cinématographiques et télévisuelles, se trouve facilité par les techniques numériques et à l'heure où se multiplient les images produites par les acteurs sociaux ordinaires au gré de leur expérience avec les caméras de leur smartphone et au gré des opportunités d'éditorialisation suivant des modes de diffusion confiés à d'autres professionnels de l'audience que les industries culturelles (blog, réseaux sociaux...). Mais comment mettre la fiction au travail pour enrichir la recherche en sciences sociales par de nouveaux usages de son pouvoir d'illusion et sans l'artificialiser?

\section{Formation à la recherche et images : un lien à développer}

4 Parallèlement, les pédagogies de l'enquête de terrain en sciences sociales se trouvent tentées d'exploiter la capacité des images à provoquer des situations d'expérience en proposant des formes d'enquête par procuration dans des milieux particuliers ou à l'intersection de plusieurs mondes sociaux (Peneff, 2009, chap. 8). Là encore, l'attention va d'abord aux productions documentaires. Une certaine proximité du regard de l'ethnographe avec celui du cinéma direct dans son projet naturaliste de rendre la richesse des situations qu'il étudie autorise cet usage (Piault, 2000), comme dans les cas de Wiseman au bureau d'aide sociale de Welfare (1975), de Louis Malle sur la chaîne automobile de Humain, trop humain (1974) ou de Depardon-Nougaret au tribunal correctionnel $(1994,1999,2004)$. A minima, la caméra permet de ramener «le terrain » dans la salle de classe et de le démultiplier. Le film offre ensuite la possibilité d'être visionné plusieurs fois pour apprendre à écarter son regard face au réel, à envisager les différents points de vue en présence, à cultiver sa mémoire, à enrichir ses notes descriptives en comparant ses performances d'observation à chaque visionnage. Cette répétition invite aussi à caractériser ce que l'œil, à l'instar de la caméra, permet de capter sur le terrain et ce sur quoi il attire spontanément l'attention, ce qu'il invite à mettre en avant. Premier mouvement dont il faut savoir se défier pour bien inscrire l'action dans son contexte de possibilité.

5 La projection du film peut être interrompue à tout moment pour discuter les choix d'orientation qui sont donnés à l'investigation documentaire et qui pourraient être donnés à une investigation résolument ethnographique. En effet, parfois indifférente à certains éléments structurants de la situation, préoccupée par la narration qui servira l'émotion qu'elle vise, la proposition documentaire prête volontiers à discussion critique, elle aussi féconde en matière de formation des apprentis chercheurs à l'enquête de terrain où ces défis de présentation se retrouvent. La comparaison du 
travail ethnographique et du travail documentaire suggère d'interroger aussi les mécaniques d'enrôlement des enquêtés et des filmés dans la coopération avec le chercheur-cinéaste (Lallier, 2011) et, notamment, d'examiner le rôle que jouent l'introduction de la caméra, l'exposition orale du projet de connaissance, la formulation de l'intention de compréhension. Produisent-elles des effets de censure par crainte des usages qui pourraient être faits des propos contre leur auteur, par sentiment d'illégitimité, par doute sur la capacité à se faire comprendre ? Ou suscitent-elles des phénomènes d'attraction par besoin de reconnaissance, par curiosité, par plaisir du jeu ? On parle parfois de profilmie (De France, 1989).

6 Mais la forme documentaire détient-elle un avantage intrinsèque sur la fiction pour être mobilisée dans ces configurations de réflexion formatrice sur les démarches d'investigation du social? Ou faut-il savoir profiter de la fluidité de la fiction dans l'approche du réel ? L'exemple de réalisateurs s'étant frottés à la fois au documentaire et à la fiction sur un même terrain, comme Claire Simon à l'occasion de son travail sur la gare du Nord ${ }^{1}$, montre que la narration fictionnelle prend une dimension très forte dans le film documentaire, par exemple pour rendre compte de la confrontation de la cinéaste à l'imaginaire fantastique lié à la foule, aux passages incessants et à l'idée que se rejoue dans ce lieu tous les jours le destin de millions de personnes. La fiction peut ensuite fournir des illustrations détaillées qui sont utiles pour comparer différents exercices professionnels de l'observation directe : celui du policier infiltré avec Donnie Brasco, celui de l'agent secret du Bureau des légendes, celui du journaliste à la Günther Wallraff dans The Man Inside. Avec pour enjeu de mieux situer le métier d'ethnographe, tirant parti d'une expérience partagée, mais à distance de l'héroïsme qui centre à l'excès l'attention sur soi.

7 Ainsi, la mobilisation de l'image dans la recherche et dans la formation à la recherche pour les disciplines des sciences sociales peut-elle sortir de la référence exclusive au film ethnologique et au cinéma documentaire, et y aurait-il quelque chose à gagner à envisager les images de fiction du cinéma et de la télévision, voire du Web quand il héberge des séquences d'autofiction produites par les acteurs sociaux au moyen de leur smartphone?

\section{Ce que dit et ce que tait la fiction}

On l'a dit, les films de fiction, au titre de productions symboliques d'une société, éclairent sa dynamique et ses particularités (Sorlin, 2015). Ils informent sur les préoccupations et sur les peurs présentes, ainsi que sur les anticipations négatives en matière d'évolution de la société ou des régimes politiques, notamment par le registre de la dystopie. Ils donnent aussi à voir une modalité de socialisation, de diffusion discrète de références pour agir en société, notamment dans les films qui jouent le réalisme et qui sont vus par une large partie de la population. Les films policiers construisent ainsi un rapport partagé à la police, à la justice et, au-delà, à la ségrégation sociale qui sert d'arrière-plan à leurs intrigues. D'autres sont pris pour étendards par des groupes particuliers: comme Philadelphia ou Les Nuits fauves pour les milieux gays des années 1990, 120 battements par minute pour les années 2010, comme Erin Brokovitch ou Promised land pour les milieux écologistes, comme La Haine ou Les Misérables pour les jeunes des quartiers d'habitat social. Ils attirent l'attention sur des pans de réalité ordinaire qui sont vus comme assumables à tel moment (les noirs et la drogue, les 
politiques et la corruption dans la série The Wire) et nous éloignent d'autres à garder invisibles, car indignes, comme l'a montré Jean Peneff à propos d'Urgences qui donne sur un mode réaliste une image bien décalée des réalités de l'hôpital de Chicago, pourtant pris pour référence explicite dans la série (1998). La réalisation de la série Hippocrate dans les années 2010 témoigne que certains sont devenus partageables: autour de la pénurie de moyens à l'hôpital sous le système de tarification à l'activité ou de la juridicisation des erreurs médicales, et d'autres toujours pas: du côté, par exemple, de la prise en charge de la saleté et de l'odeur des corps souillés. La question de l'expression culturelle n'épuise sans doute pourtant pas tous les usages possibles de la fiction cinématographique dans la recherche et dans la formation à la recherche en sciences sociales. Et l'on peut élargir l'inventaire.

\section{Comment la fiction peut sauver l'ethnographe de la contestation de son travail par ses enquêtés}

9 Pour les chercheurs en sciences sociales travaillant avec une caméra, deux problèmes au moins amplifient ce qu'Olivier Schwartz (2011) appelle, avec d'autres, le paradoxe de l'observation ethnographique qui veut qu'en présence de l'observateur, l'observé infléchisse sa conduite par rapport à ce qu'elle est d'ordinaire. L'enquêté peut refuser de laisser voir certaines pratiques par crainte de ne pas contrôler l'usage qui pourra être fait des images (pour le chercheur, il est impossible par essence de lui assurer la même protection quant à l'anonymat qu'avec l'écriture textuelle) ou, à l'inverse, en étant fasciné par le fait d'être filmé, il peut conformer exagérément sa conduite aux attentes qu'il prête à l'enquêteur. Ces deux travers partagent d'exposer le chercheur à une altération du réel et au risque de reporter cet artefact vers le spectateur de ces images. L'article de Nicola Mai dans ce volume développe une solution filmique contre ce risque en recourant à des comédiens pour rejouer le réel qui a été observé par le chercheur au moment où il travaillait sans caméra. Et rien n'interdit dans l'exemple cité de remobiliser pour cet exercice de cinéma des enquêtées sous le statut de comédiennes : sous réserve d'un mode de filmage attentif qui permette d'atteindre une justesse du jeu, comme en témoigne, dans la rubrique Un œil une image, l'expérience de Clément Dorival, producteur d'un des films de Nic Mai.

Quant au projet de narration filmique d'une investigation ethnographique menée caméra en main de bout en bout, met-il à l'abri de réactions critiques des personnes filmées? Ne doit-on pas redouter des formes d'indignation pour trahison devant l'opération de montage d'images captées à leur côté, ou des protestations devant les risques auxquels les expose toute publicisation de pratiques à la marge de la légalité ou de la légitimité sociale ? Et, là encore, le recours à des comédiens à tel ou tel moment du processus pourrait-il produire des « ethnofictions » bienvenues pour la compréhension du social, selon l'expression de Jean Rouch (Colleyn, 2009) ? C'est un peu ce qu'a proposé, peut-être à son corps défendant, Christophe Lamotte à l'occasion de la réalisation de son film Nord Paradis (2009), difficile à classer entre documentaire et fiction, éventuellement même à ranger comme exercice de sociologie d'enquête, conduit par une apprentie sociologue qui est saisie dans l'action (cf. le grand entretien de ce numéro). 


\section{Comment la fiction audiovisuelle imprègne les modes de présentation de soi et peut servir à les révéler}

11 Des films de fiction de plus en plus nombreux et affûtés sont produits par une industrie $\mathrm{du}$ divertissement de plus en plus habile en réalisme pour répondre à une consommation de plus en plus exigeante. Par-là, avec des consommations croissantes de films sur des écrans et dans des temps sociaux très variés, la linéarité de la narration des fictions cinématographiques se diffuse comme paradigme général dans nos sociétés et imprègne jusqu'au mode de présentation de l'information journalistique (storytelling), de la communication politique, de la promotion commerciale ou de la mise en scène de soi sur les réseaux sociaux (Salmon, 2007). On peut se demander comment les acteurs sociaux intègrent à leurs références pour agir ces répertoires de l'expression fictionnelle quand ils ont à se raconter dans différentes circonstances comme l'entretien d'embauche, la défense de soi devant la justice ou l'édification de ses proches par des leçons de vie. Grégory Cohen l'explore à l'occasion d'investigations sur les relations entre garçons et filles à l'adolescence et sur le répertoire des conquêtes amoureuses dans des cités d'habitat social à partir d'un dispositif d'atelier vidéo. Sophie Gebeil le regarde chez des acteurs sociaux qui travaillent à se forger sinon une identité au moins une représentation comme membres de groupes plus larges dans le cas de communautés issues de la migration qui utilisent le caractère édifiant des témoignages d'événements structurant de l'expérience comme le drame du 17 octobre 1961, tel qu'ils sont mis en forme dans des documentaires interactifs disponibles sur Internet et disposés pour fabriquer une mémoire partagée.

\section{Comment la fiction réaliste illustre l'ambition heuristique des sciences sociales et déclenche l'envie de l'alimenter dans certains cadres de visionnage}

Une proximité a été identifiée de longue date entre analyse sociologique et écriture romanesque (Lepenies, 1990) autour de la description des interactions sociales et de leur cadre, autour du récit de pratiques sociales dans leur dimension d'expérience. La question a rebondi quand, d'une part, les sciences sociales ont pris le tournant ethnographique dans leur ambition de description dense et le tournant narratif dans leur prétention à rendre compte de déterminismes qui n'écrasent pas toute marge d'action chez les acteurs sociaux ${ }^{2}$, et quand, d'autre part, la fiction littéraire s'est vue augmentée dans sa capacité descriptive par le recours à la caméra et à l'image animée et dialoguée (avec des effets de réel tenant au cadrage, au montage...). Il n'est que de constater l'apparition d'un genre en soi dans les productions des sciences sociales: le commentaire de films réalistes à caractère social, pour lequel différentes revues ont ouvert des rubriques ("Champs et contrechamps" à La Nouvelle Revue du travail, "Cinéma» dans Études. Revue de culture contemporaine, "Critiques cinéma " dans le carnet de recherche Images du travail, Travail des images). La contribution de Laurence Ellena propose de questionner cet intertexte cinématographique de la sociologie, les bonnes raisons pour lesquelles les sociologues peuvent se référer à la fiction et comment la narration les amène à s'interroger sur la formulation de la connaissance. 
Cela anime le débat sur la prétention des sciences sociales à rendre compte de configurations singulières, autant que la formation à l'enquête ethnographique s'en trouve enrichie par de nouveaux terrains, signalés ainsi à la communauté des enseignants comme investigués par procuration et partageables avec des étudiants. Il n'y a donc rien d'étonnant à ce que des projections d'images de fiction à l'occasion d'exposés de recherche où Valérie Feschet cherchait à illustrer son projet d'anthropologie des secrets de famille aient déclenché chez certains spectateurs l'envie d'échanger avec elle pour lui apporter leur concours, leur relais dans l'accès à des terrains de l'intime, dans une sorte de catharsis ethnographique.

\section{Comment la fiction audiovisuelle enrichit les répertoires de l'entretien pour accéder aux pratiques des interviewés}

Dans l'ordre de l'autoconfrontation filmique, que pourrait apporter le fait de recourir non à des images des enquêtés produites par le chercheur durant son investigation, mais à des images tournées par d'autres dans le même univers, où les personnes filmées constituent des sortes d'homologues des enquêtés qui peuvent se situer par rapport à elles au moment de commenter leurs actions? Quand Hervé Le Roux le fait avec brio à partir d'images documentaires dans son film Reprise (1997), mobilise-t-il, pour leur écho à l'expérience des interviewés et des spectateurs, la vérité du moment saisi telle qu'elle est attestée par le plan séquence de neuf minutes de La Reprise du travail aux usines Wonder qui lui sert d'appui ? Ou fait-il aussi appel à la dimension romanesque et tragique de la jeune femme qui est au centre de l'interaction et qui refuse de réintégrer l'usine? En tentant de répondre à cette question, Pascal Cesaro et Pierre Fournier prolongent un travail de recherche mené à partir d'un feuilleton des années 1960 dans le monde nucléaire (Cesaro, Fournier, 2015) et se demandent quelle part de fiction contiennent les images de Jocelyne pour constituer une ressource capable de stimuler la parole d'interviewés sur leur expérience de mai 68. Peut-on imaginer d'investiguer des questions très controversées (on pense à des sujets comme la guerre d'Algérie, la sexualité à l'heure du SIDA, l'intégration des migrants...) avec des démarches de vidéoélicitation prenant appui sur des images de fiction réaliste pour inciter les enquêtés à expliciter leurs pratiques à hauteur d'homme, telles que mises en œuvre dans des situations d'interaction sociale? 
Image 2. Un témoin et Hervé Le Roux regardent La Reprise du travail aux usines Wonder

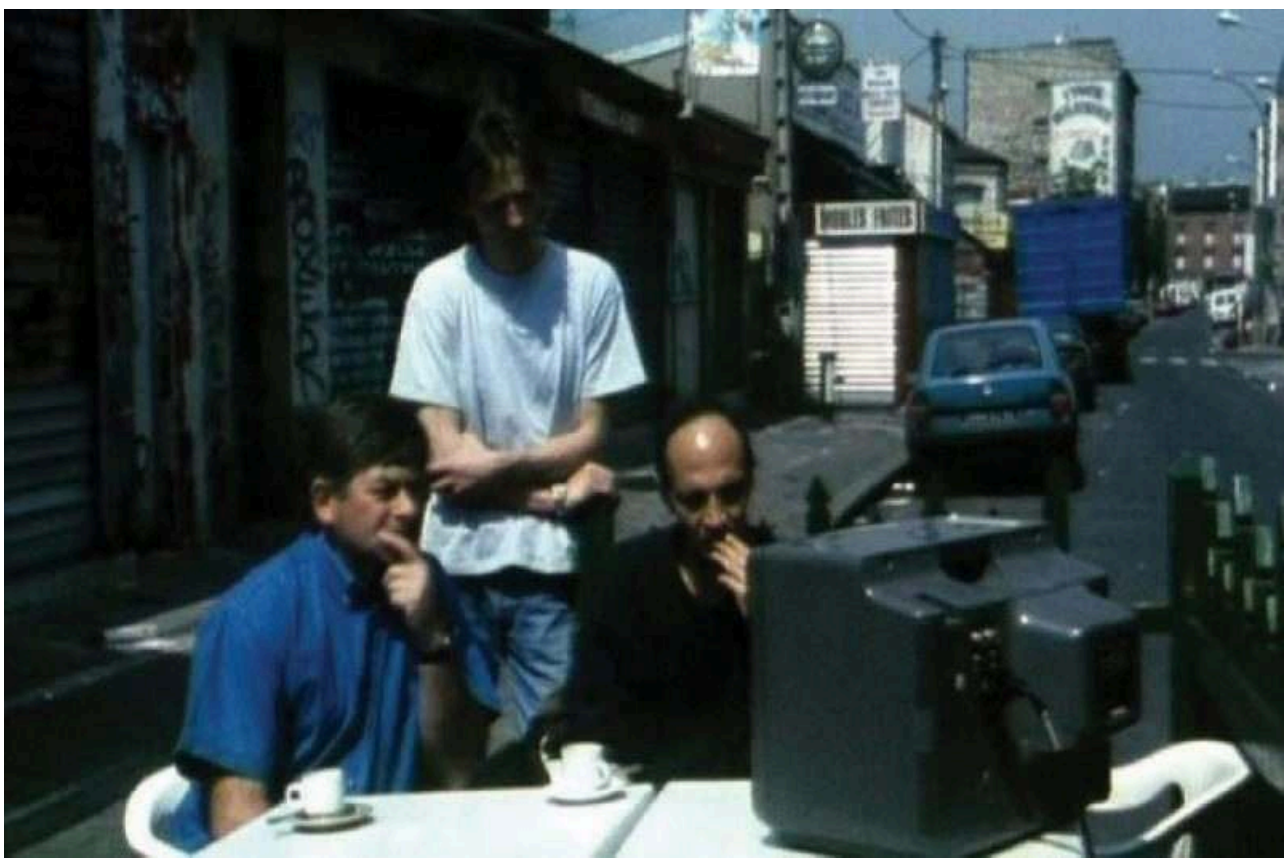

Photogramme extrait de Reprise, un film d'Hervé Le Roux (1997), copie d'écran

Ce média ne peut être affiché ici. Veuillez vous reporter à l'édition en ligne http:// journals.openedition.org/itti/1533

\section{Comment la fiction peut être mobilisée par les chercheurs dans le cadre du droit protégeant les œuvres}

L'urgence qu'il y a à proposer aujourd'hui à l'agenda des chercheurs en sciences sociales cette question des façons de mettre au travail la fiction tient à la révolution numérique qui accroît la disponibilité d'images du cinéma, de la télévision ou du web, qui facilite leur indexation, autant qu'elle simplifie l'utilisation d'outils de montage et d'hybridation dans des écritures multimédias. Elle fluidifie le dialogue avec les professionnels de l'image pour envisager des réemplois d'images dans la recherche, dans la production de connaissances nouvelles comme dans leur diffusion. Toutefois, ces pratiques posent des problèmes de droit d'auteur et de droits voisins pour les images de fiction quand elles ont été produites dans une logique économique que la recherche et la formation à la recherche ne partagent pas. La contribution de Philippe Mouron à l'éclairage de cette question recense les possibilités actuelles, et notamment les régimes d'exception pour la recherche et l'enseignement, que prévoient les textes et la jurisprudence, et pointe ce qui est de nature à les faire évoluer dans le sens d'une plus grande liberté d'utilisation des images de fiction dans la recherche et dans la formation à la recherche. 


\section{Références bibliographiques indicatives}

Beaud S. (2018) La France des Belhoumi. Portraits de famille (1977-2017), Paris, La Découverte, 352 p.

Cesaro P. et Fournier P. (2016) « De la fiction faire science : mobiliser un feuilleton télévisé des années 1960 pour parler autrement du travail dans le nucléaire ", Images du travail, travail des images [En ligne], 1 | 2016, mis en ligne le 01 février 2016, consulté le 14 février 2021. URL : http:// edt-journals.openedition.org/itti/1381

Colleyn J.-P. (dir.) (2009), Jean Rouch. Cinéma et anthropologie. Paris, Cahiers du cinéma-INA, 189 p.

France C. de [1982] (1989), Cinéma et anthropologie, Paris, Éditions de la Maison des sciences de l'Homme.

Friedmann D. (2006) «Le film, l'écrit et la recherche », Communications, 80, Filmer, chercher, sous la direction de Daniel Friedmann, p. 5-18.

Henley P. (2011) « Le récit dans le film ethnographique », L’Homme, 198-199, p. 131-157.

Lallier C. (2011) «L'observation filmante. Une catégorie de l'enquête ethnographique », L'Homme, 198-199, p. 105-130 : https://journals.openedition.org/lhomme/22718\#tocto1n4

Latour É. De (2006) « "Voir dans l'objet" : documentaire, fiction, anthropologie », Communications, 80, p. 183-198.

Lepenies W. (1990) Les Trois Cultures. Entre science et littérature, l'avènement de la sociologie, Paris, Éditions de la Maison des sciences de l'Homme, $408 \mathrm{p}$.

Peneff J. (2009) Le Goût de l'observation. Comprendre et pratiquer l'observation participante en sciences sociales, Paris, La Découverte, «Grands repères ", $254 \mathrm{p}$.

Peneff J. (1998) « La face cachée d'Urgences, le feuilleton de la télévision », Genèses, Sciences sociales et histoire, 30, p. 122-145.

Piault M.-H. (2000) Anthropologie et cinéma. Passage à l'image, passage par l'image, Paris, Nathan, $285 \mathrm{p}$.

Schwartz O. (2011) "L'empirisme irréductible : la fin de l'empirisme ? ", in Anderson Nels, Le Hobo. Sociologie du sans-abri, Paris, Colin, p. 335-384.

Salmon C. (2008) Storytelling. La Machine à fabriquer des histoires et à formater les esprits, Paris, La Découverte, $252 \mathrm{p}$.

Sorlin P. (2015) Introduction à une sociologie du cinéma, Paris, Klincksieck, 246 p.

Theureau J. (2010) « Les entretiens d'autoconfrontation et de remise en situation par les traces matérielles et le programme de recherche "cours d'action" ", Revue d'anthropologie des connaissances, 4-2, p. 287-322. 


\section{Références filmographiques}

\section{Documentaire}

$10^{e}$ chambre, instants d'audience, Depardon (2004)

Délits flagrants, Depardon (1994)

Géographie humaine, Claire Simon (2012)

Humain, trop humain, Louis Malle (1974)

La Cour des murmures, Grégory Cohen (2017)

La Cravate, Mathias Théry et Etienne Chaillou (2019)

La Punition, Jean Rouch (1962)

La Reprise du travail aux usines Wonder, Jacques Willemont (1968)

Nanook of the North, Robert Flaherty (1922)

Nord Paradis, Christophe Lamotte (2009)

Reprise, Hervé Le Roux (1997)

Welfare, Frederick Wiseman (1975)

\section{Fiction}

120 battements par minute, Robin Campillo (2017)

Donnie Brasco, Mike Newell (1997)

Erin Brockovich. Seule contre tous, Steven Soderbergh (2000)

Gare du Nord, Claire Simon (2013)

L'Affaire Wallraff, Bobby Roth (1990)

La Haine, Mathieu Kassovitz (1995)

Les Nuits fauves, Cyril Collard (1992)

Les Misérables, Ladj Ly (2019)

Philadelphia, Jonathan Demme (1993)

Promised land, Gus Van Sant (2012)

\section{Série}

Hippocrate, Thomas Lilti (depuis 2018)

Le Bureau des légendes, Éric Rochant (depuis 2015)

The Wire, David Simon (2002-2008)

Urgences, Michael Crichton (1994-2009) 


\section{Web}

Garedunord.net, un film interactif de Claire Simon (2013)

\section{NOTES}

1. Avec la fiction Gare du Nord où Reda Kateb joue un sociologue préparant sa thèse sur ce territoire et y servant de guide au personnage joué par Nicole Garcia et au spectateur, avec le documentaire Géographie humaine où la cinéaste introduit un dispositif de mise en scène justifiant que la caméra suive son ami Simon Mirabet à la rencontre de celles et ceux qui acceptent de témoigner leur vie, et avec le documentaire interactif garedunord.net où se côtoient des images de la fiction et du documentaire.

2. Le dernier ouvrage de Stéphane Beaud, La France des Belhoumi (2018), en est un formidable exemple à travers la diversité relative des parcours que rassemble la fratrie décrite.

\section{AUTEURS}

\section{PASCAL CESARO}

Pascal Cesaro est maître de conférences en cinéma à l'université d'Aix-Marseille, responsable du master Cinéma et audiovisuel et réalisateur de plusieurs films. Il enseigne la théorie et la pratique de la réalisation documentaire. Chercheur au laboratoire Perception Représentations Image Son Musique (PRISM, UMR 7061, Aix-Marseille université-CNRS), il a soutenu en 2008 une thèse intitulée Le documentaire comme mode de production d'une connaissance partagée. Ses recherches interrogent l'usage du film comme outil de recherche en sciences humaines et sociales et ses activités se développent à travers des projets de recherche-création sur les pratiques cinématographiques qu'on peut qualifier de collaboratives et sur les manières de filmer le travail, notamment dans l'univers des soins palliatifs.

\section{PIERRE FOURNIER}

Pierre Fournier est professeur de sociologie à l'université d'Aix-Marseille. Il enseigne la sociologie du travail et forme les étudiants à la pratique de l'enquête de terrain en sociologie. Au Centre méditerranéen de sociologie, de science politique et d'histoire (MESOPOLHIS, UMR 7064, Aix-Marseille université-CNRS-Sciences Po Aix), il mène des recherches sur les industries à risques (chimie, pharmacie, énergie) et leurs territoires et, au-delà, sur les enjeux de stratification sociale que portent les mots d'ordre de défense de l'environnement. Ces travaux se caractérisent par l'articulation des savoirs de la sociologie, de l'histoire, de l'économie et de l'ethnologie, ainsi que par l'usage combiné de méthodes variées : dépouillement d'archives, entretiens, traitement de données quantifiées, observation directe. 